\title{
Editorial
}

\section{Biomechanical Constitutive Model Identification}

\author{
Guillermo Rus $\mathbb{D}^{1,},{ }^{1,2,3}$ Juan Melchor $\mathbb{D}^{1,2,3}$ Marie Muller, ${ }^{4}$ and Akhtar A. Khan ${ }^{5}$ \\ ${ }^{1}$ Department of Structural Mechanics, University of Granada, Granada, 18071, Spain \\ ${ }^{2}$ Instituto de Investigación Biosanitaria, ibs. GRANADA, Spain \\ ${ }^{3}$ Excellence Research Unit "ModelingNature" (MNat), University of Granada, 18071, Spain \\ ${ }^{4}$ Department of Mechanical and Aerospace Engineering, NC State University, NC, USA \\ ${ }^{5}$ Center for Applied Computational Mathematics, Rochester Institute of Technology, NY, USA
}

Correspondence should be addressed to Guillermo Rus; grus@ugr.es

Received 26 June 2019; Accepted 26 June 2019; Published 14 July 2019

Copyright (c) 2019 Guillermo Rus et al. This is an open access article distributed under the Creative Commons Attribution License, which permits unrestricted use, distribution, and reproduction in any medium, provided the original work is properly cited.

In biomechanics, reliable modeling and quantification of material parameters and the study of constitutive models and computational tools concerning experimental observations of living tissue are a rapidly expanding field of research. The primary reason for this interest is the ever-growing number of real-world applications such as cardiovascular, osteoarticular, reproductive, osteoarticular, and regenerative biomechanics. In summary, the capability of biomathematical models to realistically predict the various behavioral patterns observed in soft tissue is a longstanding challenging problem. To advance towards this quest, this special issue presents recent developments in the topics including soft tissue biomechanical models, applied, computational, or theoretical tissue mechanics continuum field theory, hyperelastic and viscoelastic quasi-incompressible transversely isotropic constitutive model formulation and identification, inverse problems, or biomechanical parameter identification problems.

The special issue contains eleven thoroughly reviewed research papers of high scientific merit. A summary of the key ideas of the included articles is as follows.

J. Qiu and F.-F. Li proposed a methodology to determine the viscoelastic parameters for a single cell combining flow chamber experiment and fluid-structure coupled finite element model. For the optimal parameters, they used the experimental observation to compare with the deformation response of the cell with different parameters in the finite element model. The developed framework was applied to osteocytes. J. Melchor et al. assessed the mechanical properties of two groups of porcine carotid blood vessels, namely, native arteries and decellularized arteries. The biomechanical properties of both groups were determined by conducting uniaxial and circumferential tensile tests by using an ad hoc and lab-made device. T. Cui et al. developed a robotassisted craniomaxillofacial surgery system and evaluated the feasibility and reliability of this robot system in phantom experiments. J. Torres et al. explored aspects of the remodeling of the cervix, which play a fundamental role in preventing preterm birth. The authors developed a framework where the variation of the histological properties is linked with the individual biomechanical evolution of the constituents, consequently providing the feasibility of a multiscale approach. Y. Mei and P. Yu focused on characterizing heterogeneous elastic property distribution of soft tissues which has direct implication in disease detection. They proposed an inverse approach to map the heterogeneous material property distribution of soft solids using harmonic motion data. Inspired by the challenges associated with characterizing the soft tissue mechanical functionality by current elastography techniques, G. Rus et al. proposed a mechanism to evaluate the robustness of the mechanical characterization of the cervix through the torsional wave elastography probe, specifically against some relevant contact conditions. R. Muñoz and J. Melchor presented a new classical nonlinear model proposing a constitutive equation that separates volumetric and deviatoric effects and compared the formulation to constitutive equations with Landau constants for weak elasticity and both types of related nonlinear constants. $\mathrm{H}$. Jin et al. proposed a lateral balancing structure based on precession effect of double gyroscopes and its associated control strategy of the steering for an 
underactuated unicycle robot. They provided numerical results to demonstrate the rationality of the lateral balance structure and the feasibility of the steering control method. R. Palma and A. M Callejas presented a semianalytical solution based on Laplace transform to study the behavior of poroelastic materials in the context of the extended nonequilibrium thermodynamics. Utilizing the finite element simulation of the deformation that the liver undergoes during the breathing process, S. Martínez-Sanchis et al. developed a method to estimate, in vivo, the relative stiffness between a hepatic lesion and the liver parenchyma. M. S. Commisso et al. explored how the change in stress distributions affects the remodeling response of the mandible bone and showed the crucial effect of the temporomandibular joint on the stress distribution during the masticatory cycle.

\section{Conflicts of Interest}

The authors declare that they have no conflicts of interest.

\section{Acknowledgments}

Finally, we give our most sincere thanks to all the authors who have contributed to this special issue and to the collegial team of the coeditors that functioned very well together.

Guillermo Rus Juan Melchor Marie Muller Akhtar A. Khan 


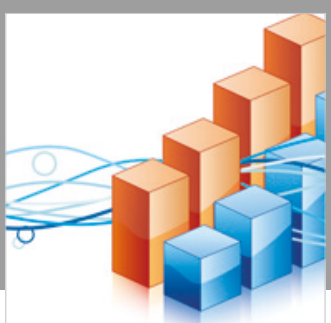

Advances in

Operations Research

\section{-n-m}
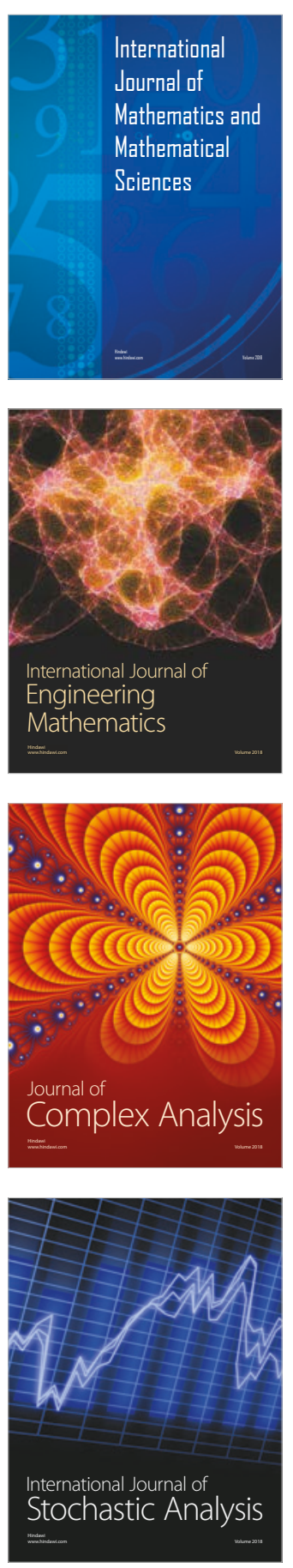
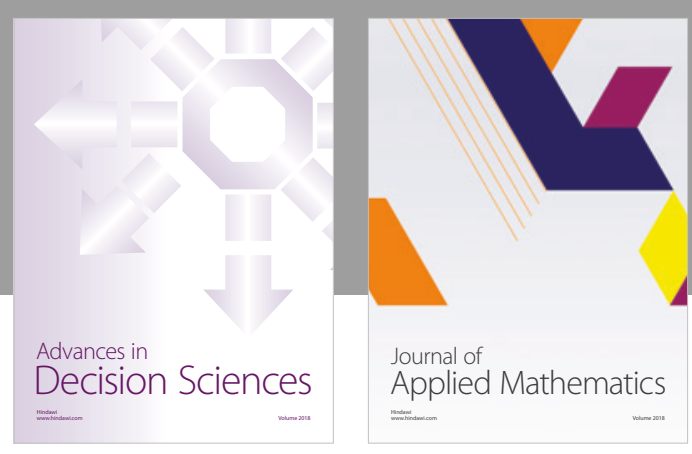

Journal of

Applied Mathematics
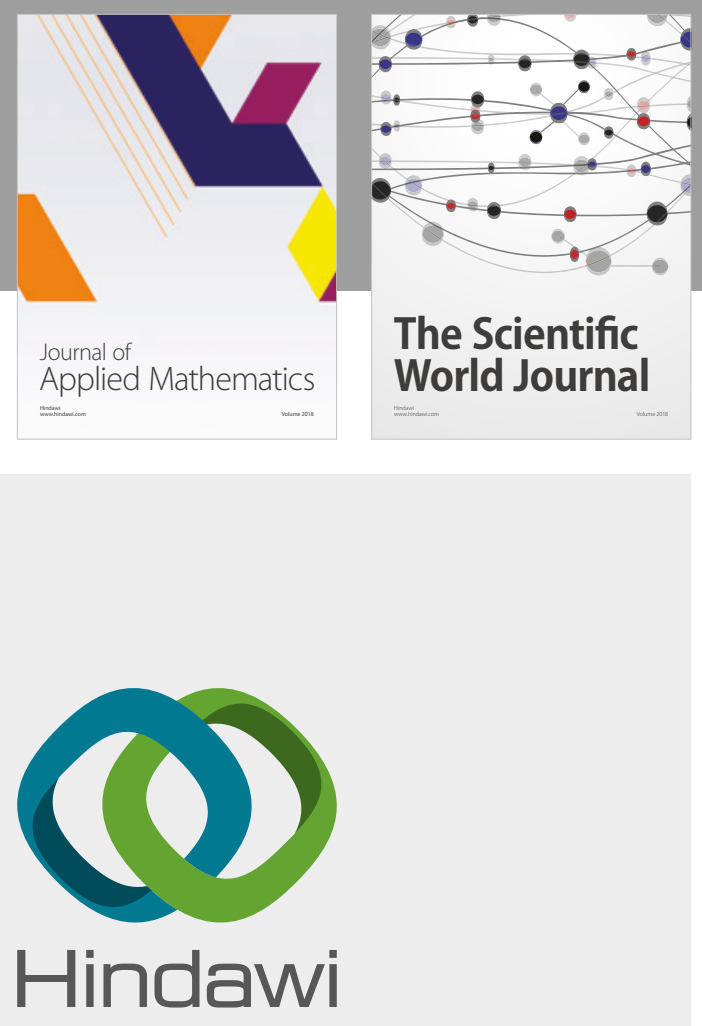

Submit your manuscripts at

www.hindawi.com

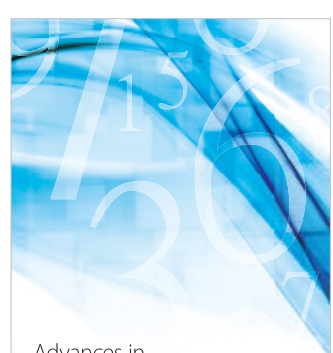

Advances in
Numerical Analysis
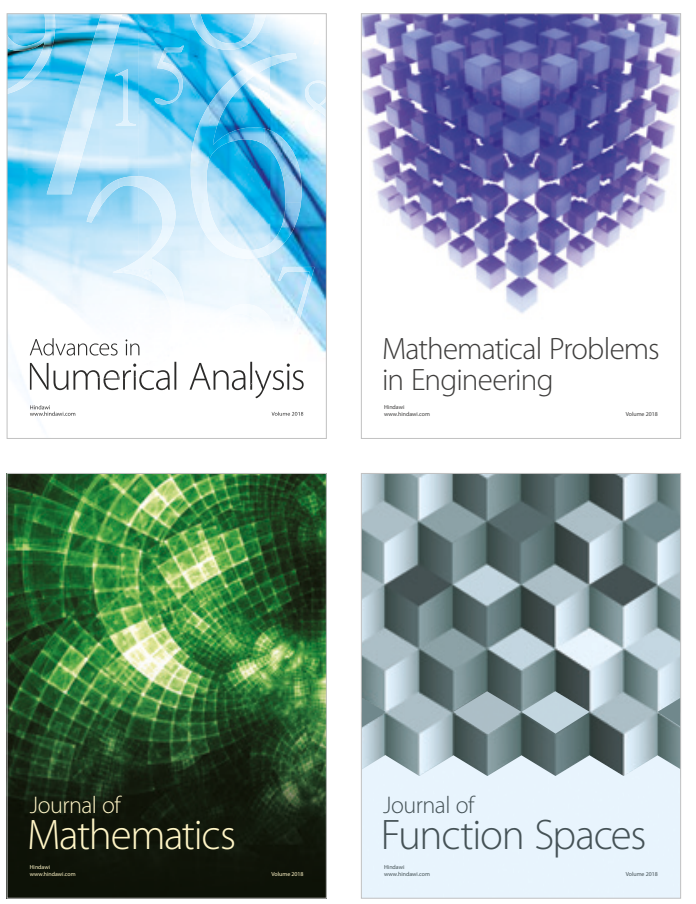

Mathematical Problems in Engineering

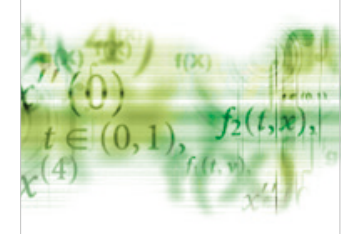

International Journal of

Differential Equations

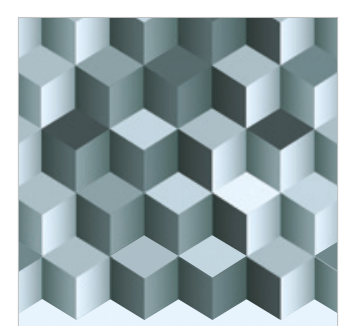

Journal of

Function Spaces

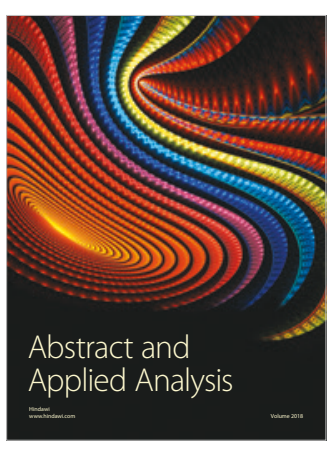

The Scientific

World Journal

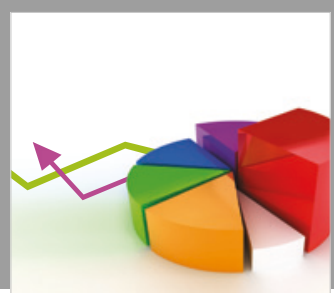

Journal of

Probability and Statistics
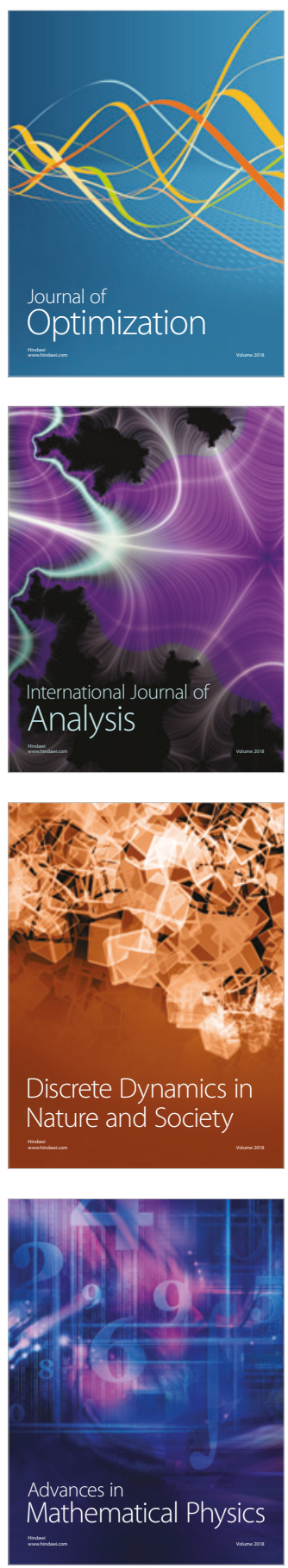